\title{
Modelo de teleducação sobre cuidados com a saúde oral do idoso usuário de prótese total dental
}

Érika Sequeira*, Rosângela Suetugo Chao**, Maria José Terra Moraes***, Vanessa Krunfli Haddad $* * * *$, Chao Lung Wen*****.

* Cirurgiã-dentista. Doutora em Ciências pela Faculdade de Medicina da Universidade de São Paulo.

** Cirurgiã-dentista. Pesquisadora colaboradora pela Disciplina de Telemedicina da Faculdade de Medicina da USP do Projeto de Tecnologias Educacionais Interativas para potencialização da educação em saúde (CAPES/MEC).

*** Cirurgiã-dentista e especialista em Periodontia pela UNICAMP.

**** Jornalista e Coordenadora de Comunicação Digital da Disciplina de Telemedicina da Faculdade de Medicina da USP.

***** Médico, Doutor e Livre Docente pela Faculdade de Medicina da Universidade de São Paulo, Coordenador do Projeto de Tecnologias Educacionais Interativas para potencialização da educação em saúde (CAPES/MEC) e chefe da Disciplina de Telemedicina da Faculdade de Medicina da USP.

\section{RESUMO}

Foi desenvolvido, aplicado e avaliado um modelo educacional interativo para explicar sobre os cuidados com a prótese total dentária e a saúde oral dos idosos. Este modelo foi implementado na cidade de Tatuí, no interior do Estado de São Paulo, Brasil. Dois grupos (A - representantes da comunidade e B - idosos) participaram desse estudo. O Grupo A foi treinado a partir do uso de uma estratégia educacional que incluiu objetos digitais de aprendizagem, infográficos, vídeos motivacionais educacionais e um ambiente interativo na web. Após o término do curso, o Grupo A ensinou aos idosos do Grupo B. Como resultado, após 06 meses de monitoramento, 96,2\% dos participantes do Grupo A afirmaram ter um dado para melhor algum hábito de cuidado com sua boca, $84,6 \%$ ensinaram alguém sobre o que aprenderam e $65,4 \%$ realizaram atividades em suas comunidades. No Grupo B, dos idosos, 93,2\% afirmaram ter aprendido algo novo, 83,3\% disseram ter melhorado o cuidado com a saúde oral e 76,4\% acabaram igualmente ensinando alguém sobre o que aprenderam. As ferramentas digitais educacionais e os recursos de teleducação mostraram-se eficientes. Os participantes mudaram seus hábitos positivamente e melhoraram seus conhecimentos sobre cuidados com a saúde oral. A perspectiva é a aplicação destes recursos educacionais interativos e o modelo de teleducação em apoio à atenção primária, e em atividades em que se aplica a teleducação para difundir conhecimento sobre cuidados em saúde oral e atenção à saúde dos idosos.

Descritores: idoso, prótese total dentária, teleducação, higiene oral. 


\section{INTRODUÇÃO}

A população brasileira atingiu um total de 190.755.799 habitantes em 2010. Simultaneamente, pode ser observado o crescimento da população com 65 anos ou mais, considerada idosa, que era de $4,8 \%$ em 1991, passando a 5,9\% em 2000 e chegando a 7,4\% em 2010. ${ }^{3}$

No Brasil, a condição mais observada, quando se trata da saúde oral em idosos, é o edentulismo, expressão máxima da mutilação dentária $^{19}$. De acordo com a Pesquisa Nacional de Saúde Bucal, na faixa etária das pessoas com idades entre 65 e 74 anos, a necessidade de uso de prótese total dupla era de $15,4 \%$ e a necessidade de prótese total em uma arcada era $23,9 \%$ no ano de $2010 .^{2}$

A falta de dentes é prevalente nos idosos de todo o mundo e está fortemente associada com a condição socioeconômica. Estudos epidemiológicos demonstraram relação direta entre situação financeira, grau de escolaridade e edentulismo, de forma que possuir dentições naturais são mais frequentes em idosos de classes socioeconômicas altas. ${ }^{17,4,6,15,20,7}$

A perda dental reduz a capacidade mastigatória, o que pode influenciar a seleção dos alimentos, o status nutricional e a saúde geral. A manutenção de uma boa higiene oral é um importante promotor de saúde na população em envelhecimento. ${ }^{19}$

Para os idosos em que uma reabilitação protética é necessária, uma prótese bem adaptada e em boas condições é uma mantenedora da saúde geral e essencial para uma qualidade de vida saudável. ${ }^{11,9,16,10,25}$ Possuir uma saúde oral adequada possibilita ao ser humano exercer funções como mastigação, deglutição e fonação, além de exercitar a autoestima e relacionar-se socialmente sem inibição ou constrangimento. ${ }^{17}$
Muitos idosos não dão a devida atenção às medidas preventivas por muitas razões, como: percepções negativas sobre a odontologia, desmotivação ou desconhecimento sobre os tratamentos disponíveis. ${ }^{14,22}$

Idosos, com frequência, não estão devidamente informados sobre a importância da saúde e da higiene oral e não são orientados para realizá-la. ${ }^{8} \mathrm{~A}$ falta de dentes e o desconforto oral passam a ser considerados características típicas do processo de envelhecimento, sendo aceitos como consequências inevitáveis. ${ }^{23,18}$

Informação e orientação básica constituem um meio para modificação de comportamentos e possível melhoria na qualidade de vida. Portanto, a proposta desse trabalho foi desenvolver um modelo de educação interativa para capacitar pessoas a multiplicarem conhecimento em suas comunidades e avaliarem sua contribuição junto ao público idoso, em forma de mudança de opinião e comportamento.

\section{MATERIAL E MÉTODOS}

Este modelo teleducacional foi desenvolvido para capacitar representantes de uma comunidade (Grupo A) para que ensinassem idosos usuários de prótese total dentária (Grupo B) sobre a importância da saúde e da higiene oral.

A sistemática desse modelo de teleducacão interativa sobre a saúde oral dos idosos abrangeu a criação de materiais instrutivos de apoio (objetos de aprendizagem em computação gráfica 3D, um vídeo motivacional em DVD e infográfico usado em forma de folheto), e a criação de uma estratégia de comunicação para ensinar os representantes da comunidade (Grupo A) em um ambiente de teleducação com estruturação de um ambiente virtual de aprendizagem, 
no qual havia textos complementares e lista de discussão (baseada nos temas mais relevantes para a promoção de saúde oral dos idosos, sustentados pela literatura científica).

$\mathrm{O}$ vídeo motivacional incluiu filmagens e a criação de imagens provenientes de recursos de modelagem gráfica computacional em três dimensões (Projeto Homem Virtual da Disciplina de Telemedicina da Faculdade de Medicina da Universidade de São Paulo), o que transformou o conteúdo teórico em uma comunicação visual dinâmica.

O folheto, contendo os infográficos, sintetizava as etapas mais relevantes para a higiene oral da prótese total dentária, com a estratégia de apresentar letras maiores, imagens e design para facilitar a leitura pelos idosos, além de ser plastificado para uso em ambientes úmidos e manuseio frequente.

Para esta pesquisa, foi necessário um ano de planejamento e desenvolvimento dos conteúdos e materiais educacionais, seis meses para a aplicação e coleta dos dados e mais seis meses para a compilação dos dados e conclusão do estudo.

Participaram desta pesquisa residentes do município de Tatuí, no interior do estado de São Paulo - Brasil, cuja população estimada é de 106.572 pessoas. ${ }^{3}$

Em relação aos critérios de inclusão, o Grupo A foi formado por voluntários, representantes da comunidade, que trabalhassem ou que tivessem interesse em trabalhar com idosos (cuidadores), com idades variando entre 20 e 55 anos, homens ou mulheres, sem restrição quanto à escolaridade e que tivessem conhecimento básico de informática e acesso à internet. O Grupo B constituiu-se de idosos, voluntários, com idade de 60 anos ou mais, sem restrição quanto ao gênero ou nível educacional, que fossem independentes em suas atividades básicas diárias, como escovar os dentes, usar o toalete, se vestir e se alimentar.

O Grupo A recebeu treinamento sobre a saúde oral dos idosos por meio de um curso blended learning, isto é, que combinou reuniões presenciais e uso de ambiente de teleducação. Em uma primeira reunião presencial, foi apresentado como seria o curso e o uso do ambiente de teleducação. Seguiu-se então um período de 15 dias para estudo em um ambiente virtual de ensino chamado Cybertutor, envolvendo o uso das listas de discussões, chat e testes referentes aos conteúdos apresentados. Ao fim desta fase a distância, uma segunda reunião aconteceu para o esclarecimento das dúvidas e treinamento sobre o uso dos materiais instrutivos de apoio (vídeo motivacional e folheto), posteriormente entregues a cada integrante do Grupo A.

Em um dia chamado "Dia do Encontro", os integrantes do Grupo A ensinaram aos integrantes do Grupo B, dos idosos, o que haviam aprendido. Este encontro ocorreu em um Centro de Convivência de Idosos e incluiu a apresentação do vídeo motivacional, seguido por uma dinâmica em grupos para esclarecimento de dúvidas e debate sobre o que foi apresentado (cerca de 8 a 10 idosos do Grupo B para 2 ou 3 membros do Grupo A). Dentro da estratégia de comunicação, foi entregue a cada idoso um DVD e um folheto, para que pudesse rever o que aprendeu quando quisesse.

Para avaliação dos resultados, foram aplicados questionários, antes e depois da conclusão do curso via teleducação, para o Grupo A, com a finalidade de aferir o grau de motivação em ensinar aos idosos. Este mesmo grupo, após 45 dias e após 06 meses do Dia do Encontro, respondeu a novos questionários para verificar se foram realizadas outras atividades junto à comunidade. No Grupo B, foram feitas entrevistas antes 
e depois do Dia do Encontro, após 45 dias e 06 meses, para verificar se houve mudança de comportamento e de opinião, baseada na percepção dos idosos em relação à sua própria saúde oral.

Para análise estatística, foi utilizado o software SPSS (Statistical Package for the Social Sciences). Foram feitas análises descritivas dos dados, sobre as quais foram calculadas médias, desvio padrão, mínimo, mediana, máximo frequência e percentual. Foi utilizado um nível de significância de $5 \%(\mathrm{p}$-valor $\leq 0,05)$.

\section{RESULTADOS}

O conteúdo apresentado no ambiente interativo de aprendizagem (teleducação interativa), o vídeo motivacional e o folheto foram as ferramentas educativas desenvolvidas e usadas neste modelo.

Foram desenvolvidos 09 módulos, com conteúdo teórico disponibilizado em ambiente interativo de aprendizagem (denominado Cybertutor da Disciplina de Telemedicina da Faculdade de Medicina da Universidade de São Paulo - DTMFMUSP) contendo os seguintes temas: envelhecimento; próteses dentárias: cuidados com higiene e manutenção; envelhecimento oral; higiene oral; idosos: educação e comunicação; câncer de boca; mitos e verdades; para saber mais e considerações finais.

Foram desenvolvidos objetos de aprendizagem baseados em sequências dinâmicas de computação gráfica em 3D (Projeto Homem Virtual da DTM-FMUSP), abordando a higiene da prótese total dentária (PTD) e cuidados com a saúde oral, como a realização do autoexame para prevenção do câncer de boca (Figuras 1 e 2). Com o uso dessas imagens e filmagens externas (baseadas em um roteiro), foi criado um vídeo educacional com propósitos de orien- tação e motivação, com duração de 10 minutos, sobre saúde oral dos idosos e higiene das PTDs, disponibilizado em DVD.

Figura1 - Imagem do Projeto Homem Virtual DTM - FMUSP

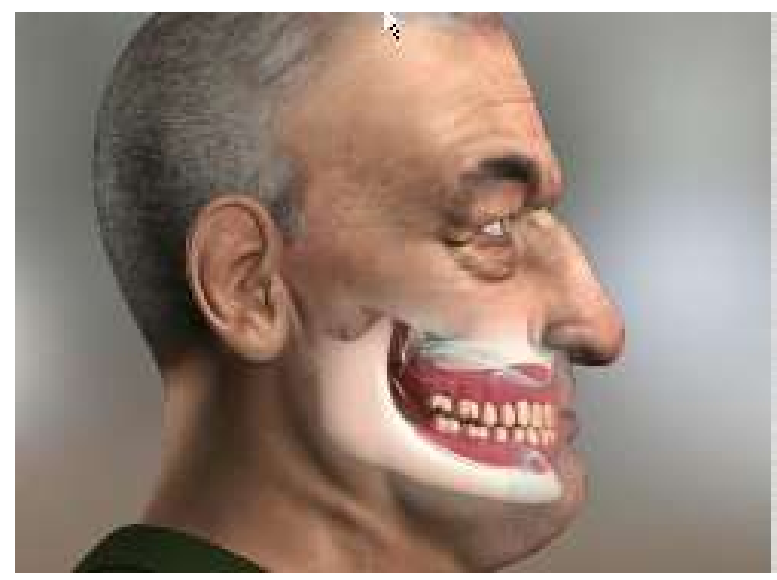

Figura 2 - Imagem do Projeto Homem Virtual DTM - FMUSP

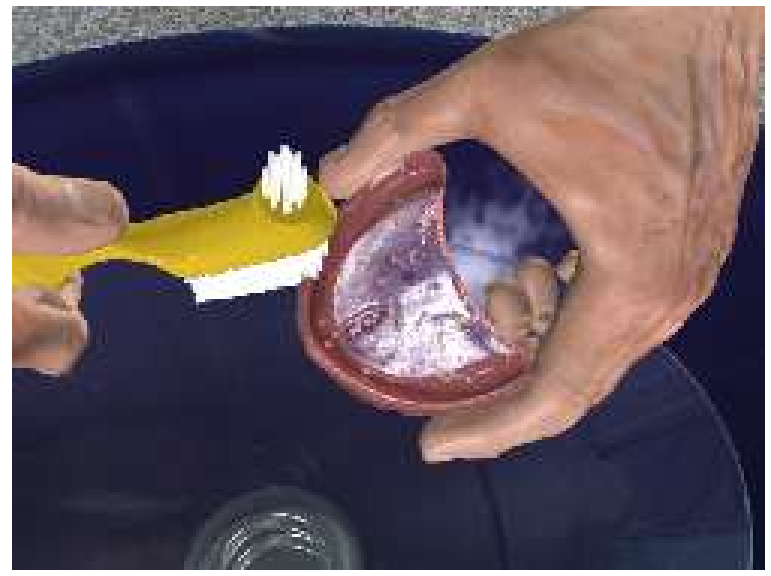

Com o uso das imagens do Projeto Homem Virtual associadas a mensagens significativas, foi elaborado um infográfico em forma de folheto, contendo 10 passos importantes para manutenção da higiene da boca e da PTD. Os passos 1 a 6 abordavam sobre a higiene da PTD, 7 sobre a higiene da boca, 8 sobre o autoexame para prevenção do câncer de boca, 9 sobre higiene da língua e 10 sobre a necessidade de o idoso procurar um profissional quando notar alguma alteração na boca ou na PTD (Figura 3). 
Figura 3 - Imagem interna do folheto explicativo

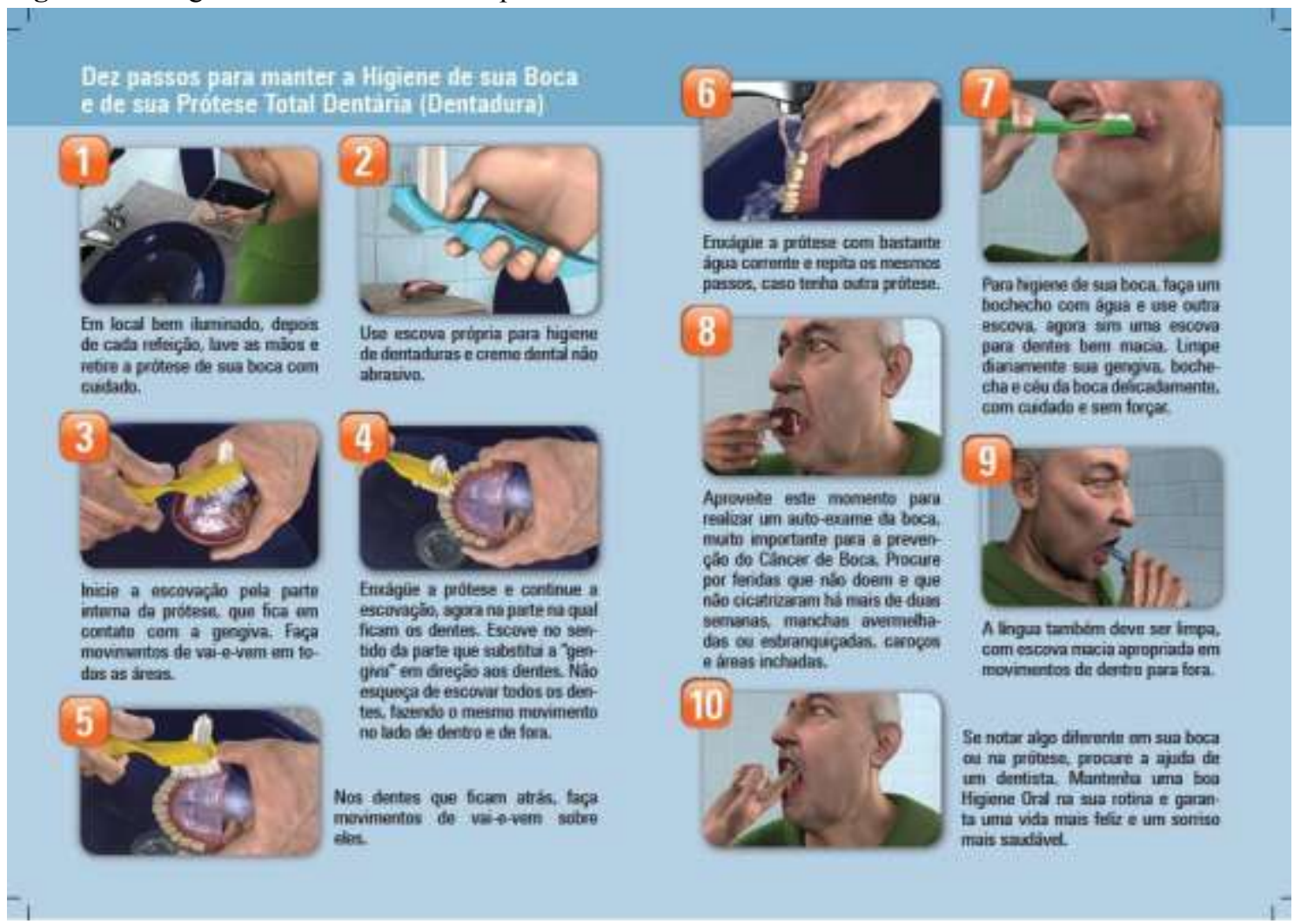

Em relação aos Grupos, no Grupo A, 26 pessoas iniciaram e completaram todas as etapas da capacitação. Destas, $92,3 \%$ eram mulheres e $7,7 \%$ homens, com idade média de 34 anos; 42\% haviam completado o ensino médio e $65,4 \%$ atuavam na área da saúde.

No Grupo B, dos idosos, de um grupo inicial com 97 indivíduos, 57 finalizaram o estudo após 06 meses. Dos 97 idosos, $77,3 \%$ eram mulheres e $22,7 \%$ homens, com idades variando de 60 a 85 (média de 69 anos). Desses, 93,8\% não completaram o ensino fundamental e 44,3\% consideravam satisfatória sua saúde geral naquele momento.

O Dia do Encontro entre os Grupos A e B durou 04 horas. Os idosos assistiram 2 vezes ao vídeo sobre saúde oral e participaram da dinâmica divididos em 08 grupos. Ainda neste dia, houve uma aula sobre o autoexame para prevenção do câncer de boca e demonstração de instrumentos para higiene oral. Ao final, receberam um kit de saúde oral, que continha um DVD com vídeo educativo motivacional, um folheto explicativo, uma escova macia para higiene da boca e uma escova própria para higiene de PTD.

No Grupo A, 92,3\% informaram que atendiam idosos em seu local de trabalho e $100 \%$ dos alunos sentiam falta de conhecimento específico sobre o processo de envelhecimento. Em relação ao ambiente de teleducação, $88,5 \%$ informaram que nunca tinham participado de outros cursos a distância.

Após a realização do curso via teleducação, 84,6\% avaliaram como ótima a qualidade das informações teóricas, 96,2\% consideraram que o curso contribuiu com conhecimento adicional e relataram que mudaram algum hábito relacionado ao cuidado com a saúde de sua boca e $92,3 \%$ confirmaram que o curso superou suas expectativas. 
Comparando os momentos após 45 dias e 6 meses do dia do encontro, a maioria dos representantes da comunidade ensinou o que aprendeu e realizou atividades em suas comunidades (Tabela 1). Além disto, após 06 meses do Dia do Encontro, $65,4 \%$ afirmaram que realizaram novas atividades em suas comunidades. Desses, $64,7 \%$ usaram como material de apoio o vídeo e $76,5 \%$ usaram o folheto explicativo.

Tabela 1 - Comparação após 45 dias e 06 meses - Grupo A

\begin{tabular}{c|cc}
\hline $\begin{array}{c}\text { Pergunta - Grupo A } \\
\text { (representantes da comunidade) }\end{array}$ & Após 45 dias & Após 06 meses \\
\hline Ensinaram alguém sobre o que aprenderam? & $96,2 \%$ & $84,6 \%$ \\
\hline Realizaram alguma atividade na comunidade? & $69,2 \%$ & $65,4 \%$ \\
\hline
\end{tabular}

No Grupo B, dos 97 idosos que iniciaram o estudo, 80,4\% eram usuários de PTD. Desses, 37,9\% relataram problemas com suas próteses e 39,3\% usavam a mesma prótese há mais de 10 anos.

Mais da metade dos usuários de PTD não receberam orientações anteriores sobre a higiene da prótese $(60,8 \%)$, higiene da boca $(64,6 \%)$, como usá-la $(62,8 \%)$ ou sobre a necessidade de se fazer retornos periódicos ao dentista (76,9\%). A grande maioria, $88,5 \%$, não fazia retornos periódi$\cos$ ao dentista, principalmente porque achava que não era mais necessário $(52,2 \%)$.

Imediatamente após o dia do encontro, $73 \%$ consideraram muito importantes as informações do vídeo e 93,2\% relataram que aprenderam algo que não sabiam.

Após 45 dias do dia do encontro: $84,4 \%$ dos idosos afirmaram que houve alguma mudança de hábito relacionada ao cuidado com a saúde oral. As mudanças mais citadas foram a higiene da prótese $(56,4 \%)$ e a higiene da língua (34,5\%). A maioria assistiu novamente ao vídeo e ensinou alguém sobre o que aprendeu. Sobre a motivação, 96,9\% afirmaram continuar motivados para cuidar melhor da saúde da boca após o dia do encontro (Tabela 2).

Após seis meses do dia do encontro: $83,3 \%$ continuaram a afirmar que houve alguma mudança de comportamento depois daquele dia em relação ao cuidado com a boca, sendo que a higiene oral e da língua foram as mais citadas; $69,6 \%$ assistiram novamente ao vídeo; $76,4 \%$ ensinaram alguém sobre o que aprenderam no dia do encontro; $89,1 \%$ leram novamente o folheto explicativo. E, em relação à motivação para cuidar melhor da saúde da boca, 91,2\% disseram que continuavam motivados (Tabela 2). 
Tabela 2 - Comparação após 45 dias e 06 meses - Grupo B

$$
\text { Pergunta - Grupo B (idosos) Após } 45 \text { dias } \quad \text { Após } 06 \text { meses }
$$

Mudança de comportamento em relação ao cuidado com sua Saúde Oral.

Assistiram ao vídeo novamente

Ensinaram alguém sobre o que aprenderam

Higiene da língua

Continuaram motivados

Leram novamente o folheto

Todos os idosos que usavam PTD passaram a fazer a higiene da dentadura com água e creme dental em substituição a outros produtos, como sabão e sabonete, e 94\% passaram a usar a escova própria para prótese para higiene da PTD.

Comparando os questionários logo após o encontro com o pós 45 dias do em contro, 64\% dos idosos que pretendiam assistir outras vezes ao vídeo realmente assistiram, 56\% dos que disseram que iriam pedir para alguém assistir ao vídeo, real- mente assistiram com alguém e $75 \%$ dos que pretendiam ensinar alguém realmente ensinaram.

Desses idosos que assistiram ao filme outras vezes, $65 \%$ fizeram o autoexame para prevenção do Câncer de Boca. Ao passo que, dentre as pessoas que não assistiram ao filme outras vezes, $35 \%$ fizeram o autoexame para prevenção do Câncer de Boca (Teste Qui-Quadrado: pvalor $=0,0205)($ Gráfico 1).

\section{Gráfico 1. Autoexame para prevenção do cancer de boca $x$} Assistiu DVD outras vezes.

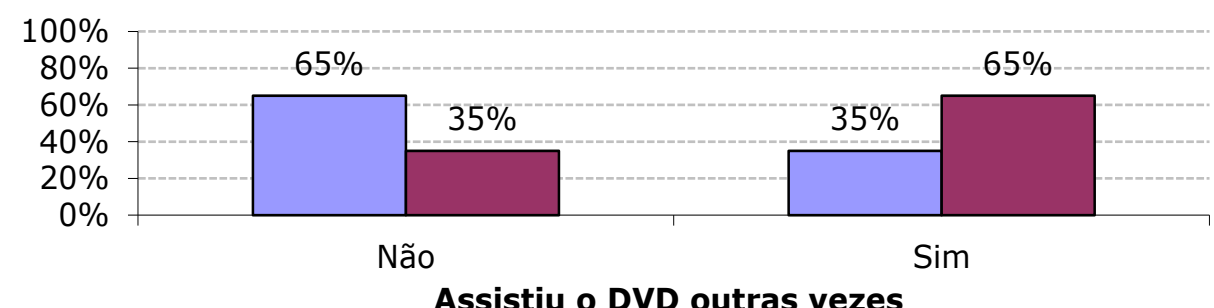

Assistiu o DVD outras vezes

$$
\text { 口Não } \square \operatorname{Sim}
$$

Em relação à frequência da realização da higiene oral, no momento pós 45 dias, $36,8 \%$ dos idosos faziam a higiene oral mais de três vezes ao dia. No momento pós 6 meses, o percentual diminuiu para $19,3 \%$. 


\section{DISCUSSÃO}

A provisão de informação e educação é um aspecto fundamental para a adoção de comportamentos saudáveis. ${ }^{13}$

Entender as necessidades dos idosos, a forma como percebem a importância da saúde e da higiene oral e estabelecer estratégias para encorajá-los a melhorar hábitos e a mudar comportamentos é fundamental.

A comunicação é um dos pontos importantes no desenvolvimento do plano de tratamento ao idoso. À medida que se envelhece, declínios sensoriais podem ser obstáculos para a comunicação. ${ }^{8}$ Alguns idosos podem simplesmente não compreender as explicações durante uma consulta ao profissional da saúde, perdendo uma oportunidade de sanar dúvidas e de melhorar seu desempenho com o autocuidado.

A educação em higiene oral e manutenção da saúde para pacientes de modo geral ocorre em consultório odontológico, por meio de uma explanação oral, sendo raros os materiais e as campanhas voltadas especificamente ao público idoso. As orientações sobre saúde oral não são fixadas como deveriam e não são absorvidas como passíveis de serem integradas ao dia a dia, devido à forma e o tempo despendido para transmitir estas informações, que são essenciais aos pacientes. ${ }^{13}$

Neste estudo, um modelo educacional interativo foi desenvolvido e aplicado em um plano de ação que permitisse sua replicação em diversas comunidades. Por meio de ferramentas acessíveis e que independam da dedicação exclusiva de um especialista, formou-se uma "Cadeia Produtiva em Saúde", na qual a própria comunidade é capacitada e se torna um agente multiplicador de informações.
Em uma "Cadeia Produtiva em Saúde", ocorre uma reorganização dos processos na saúde, na qual modernas tecnologias são usadas para promover ações integradas e multiprofissionais. Através dela, é possível desenvolver um modelo de relacionamento entre profissionais de saúde e população, no qual o enfoque é a promoção da saúde, por meio da educação, com estímulo à qualidade de vida saudável.

O curso dispõe de uma modalidade de teleducação interativa (Cybertutor), que utilizou web site para facilitar o ensino e o acesso a recursos interativos. Foi uma alternativa que facilitou o acesso aos conteúdos, mesmo que os alunos estivessem distantes fisicamente, e permitiu flexibilidade aos alunos para organizarem o seu melhor período de estudo. A teleducação interativa mostrou ser um meio de educação viável, visto que 96,2\% dos integrantes do Grupo A consideraram que o curso contribuiu com conhecimento adicional e $100 \%$ conseguiram aprender usando esta dinâmica. O conteúdo aprendido gerou mudança de hábito no cuidado com a saúde oral em 96,2\% e $69,2 \%$ cumpriram seu papel realizando atividades em suas comunidades.

Educar e capacitar não são simplesmente fornecer grandes quantidades de informações, mas sim desenvolver uma estratégia de comunicação e interação. ${ }^{24}$ Por isso, o conjunto desenvolvido (vídeo educacional e folheto) representa um método visual claro, dinâmico e motivador para se obter uma possível melhora na retenção de informações fundamentais para a manutenção da saúde oral, modernizando-se a forma de orientação e buscando amparar os idosos.

Para que fosse possível desenvolver 
um vídeo que transmitisse as informações de forma simples e clara, adequou-se o conhecimento científico para uma linguagem que fosse de fácil entendimento pelo idoso.

Como apoio didático, utilizou-se também recursos da comunicação visual através da computação gráfica 3D (Projeto Homem Virtual) para transmitir conhecimentos de forma dinâmica (objetos de aprendizagem), clara e sem constrangimento, podendo ilustrar aspectos difíceis de serem mostrados por meio da filmagem convencional, com o uso, por exemplo, de transparência em estruturas anatômicas.

A estes objetos foram agregados recursos como roteirização de assuntos com inclusão de mensagens significativas, filmagens, sonoplastia e narração para reforçar a transmissão de conhecimentos.

Diante da possibilidade de se rever o vídeo educativo quantas vezes fosse desejada, permitiu-se o acesso rápido às informações, reforçando o aprendizado $(62,5 \%$ dos idosos assistiram novamente ao filme). Tanto o vídeo como o folheto foram elaborados com foco nas necessidades do idoso, com uma linguagem clara e com o uso de mensagens significativas.

A parceria entre comunicação, educação e novas tecnologias deve se articular como suporte de uma comunicação educativa mais diversificada. ${ }^{12}$ Assim, há a constituição de equipes multidisciplinares pedagógicas para desenvolver processos educacionais. $^{21,1}$

Como resultado desta nova forma de comunicação, 93,2\% dos idosos afirmaram ter aprendido algo que não sabiam e $89 \%$ dos que pretendiam melhorar a higiene da boca relataram realmente ter melhorado.

No aspecto das mudanças de hábitos, foi constatado que: $100 \%$ dos idosos passaram a utilizar água e creme dental para higiene de suas próteses ao invés de outros produtos como desengordurantes, sabão e sabonete; $90 \%$ estavam usando uma escova para limpar a boca e outra escova exclusivamente para a higiene da prótese, o que é ideal.

A maioria dos idosos usuários de PTD relatou não ter recebido previamente nenhum tipo de orientação em relação à higiene das próteses $(60,8 \%)$, por isso as instruções de higiene devem ser enfatizadas. O momento da entrega de uma prótese total deveria ser o início de uma longa relação profissional-paciente. ${ }^{5}$

Neste estudo, $75 \%$ dos idosos relataram ter ensinado alguém sobre o que aprenderam no dia do encontro, com o vídeo e o folheto, ou seja, além do Grupo A capacitado, os próprios idosos se tornaram multiplicadores em suas famílias e comunidade.

Atividades como esta, no campo da telessaúde, adquirem efetividade quando associadas a planos estratégicos, que incluam um processo de logística de distribuição de serviços de saúde, em sincronia com os benefícios que podem trazer, e não quando simplesmente ultrapassam a fronteira da tecnologia em si.

\section{CONCLUSÕES}

Ambos os grupos melhoram o entendimento sobre a relevância de se realizar a prevenção e a manutenção da saúde oral.

O modelo educacional, baseado em teleducação interativa, possibilitou o aprendizado sobre saúde oral pelos participantes e mostrou ser uma forma de comunicação acessível, que facilitou a compreensão e retenção das informações e orientações disponibilizadas.

A perspectiva é a sua utilização em atividades de apoio à atenção primária e saúde do idoso e em atividades que utilizem 
teleducação interativa como recurso para promover treinamento e capacitação em larga abrangência.

\section{REFERÊNCIAS}

1. Alves LRG, Nova CC. Educação à distância: limites e possibilidades. In: Educação à distância: uma nova concepção de aprendizado e interatividade. Futura, São Paulo, 2003:43-50.

2. Brasil. Ministério da Saúde. Sistema Único de Saúde. Pesquisa Nacional de Saúde Bucal; 2010.

3. Brasil. Ministério do Planejamento, Orçamento e Gestão. Instituto Brasileiro de Geografia e Estatística. Censo Demográfico 2010; 2010.

4. Budtz-Jorgensen E. Prosthodontics for the Elderly: Diagnosis and Treatment. Quintessence Publishing, 1999:1-21.

5. Castellucci Barbosa L, Ferreira MR, de Carvalho Calabrich CF, Viana AC, de Lemos MC, Lauria RA. Edentulous patients knowledge of dental hygiene and care of Prostheses. Gerodontology 2008; 25:99-106.

6. Colussi CF, Freitas SFT. Aspectos epidemiológicos da saúde bucal do idoso no Brasil. Cad Saúde Pública 2002;18(5):1313-1320.

7. Colussi CF, de Freitas SF. Edentulousness and associated risk factors in a south brazilian elderly population. Gerodontology 2007;24(2):93-7.

8. Couto EAB. A comunicação com o idoso-técnicas e estratégias. In: Campostrini E. Odontogeriatria. Revinter: Rio de Janeiro, 2004:32-37.

9. Ettinger RL. Oral disease and its effect on the quality of life. Gerodontics. 1987;3:103-106.

10. Fukunaga A, Uematsu H, Sugimoto $\mathrm{K}$. Influences of aging on taste per- ception and oral somatic sensation. $\mathbf{J}$ Gerontol A Biol Sci Med Sci 2005;60:109-13.

11. Gavinha S, Braz MP, Sousa L. Odontogeriatria: conhecer para ajudar. Rev Por Clin Geral. 2006;22:391-399.

12. Gómez GO. Comunicação, Educação e novas tecnologias: tríade do século XXI. Rev Comunicação \& Educação 2002;(23):57-70.

13. Harris CE, Chestnutt IG. The use of the Internet to access oral healthrelated information by patients attending dental hygiene clinics. Int J Dent Hyg 2005;3(2):70-3.

14. Kinsey JG, Winstanley RB. Utilization of domiciliary dental services. Gerodontology 1998;15(2):107-112.

15. Manski RJ, Goodman HS, Reid BC, Macek MD. Dental Insurance Visitis and Expenditures Among Older Adults. Am J Public Health 2004;94(5):759-64.

16. Marucci MFN. Nutrição e seu papel para o envelhecimento saudável. Anais do II Encontro Interdisciplinar de Odontologia em Gerontologia. Casa do Novo Autor, São Paulo, 2000:76-84.

17. Narvai PC, Antunes JLF. Saúde bucal: a autopercepção da mutilação e das incapacidades. In: Lebrão ML, Duarte YAO. O projeto SABE no município de São Paulo: uma abordagem inicial. OPAS, Brasília, 2003:119-140.

18. Nunes CIP, Abegg C. Factors associated with oral health perception in older Brazilians. Gerodontology 2008;25:42-48.

19. Paganini-Hill A, White SC, Atchison KA. Dental Health Behaviors, Dentition, and Mortality in the Elderly: The Leisure World Cohort Study. Journal 
of Aging Research. Volume 2011, Article ID 156061, 10 pages Disponível em: [downloads.hindawi.com/journals/specialiss ues/759894.pdf].

20. Petersen PE, Yamamoto T. Improving the oral health of older people: the approach of the WHO Global Oral Health Programme. Community Dent Oral Epidemiol 2005;33(2):81-92.

21. Ramal AC, Educação à distância: entre mitos e desafios. Rev Pátio 2001;18:12-16.

22. Saliba CA, Saliba NA, Marcelino G, Moimaz SAS. Saúde Bucal dos Idosos: uma realidade ignorada. Rev APCD 1999;53(4):279-282.

23. Silva SRC, Fernandes RAC. Autopercepção das condições de saúde bucal por idosos. Rev Saúde Pública 2001;35:349-355.

24. Wen CL. Modelo de ambulatório virtual (cyber ambulatório) e tutor eletrônico (cyber tutor) para aplicação na interconsulta médica, e educação a distância mediada por tecnologia. Tese de Livre Docência. Faculdade de Medicina da Universidade de São Paulo, Departamento de Patologia, 2003.

25. World Health Organization. Oral health in ageing societies: Integration of oral health and general health. WHO Global Oral Health Programme, Geneva, 2006.

\section{AGRADECIMENTOS}

Agradecimentos ao Projeto de Telemedicina do Programa Institutos do Milênio do $\mathrm{CNPq}$ (Conselho Nacional de De- senvolvimento Científico e Tecnológico) / Ministério da Ciência e Tecnologia.

\section{ABSTRACT \\ Teleducational model about elderly oral health care with total dental prosthesis}

Was developed, applied and evaluated an interactive educational model to teach about elderly oral health and total dental prosthesis care. This model was implemented in the city of Tatuí (Sao Paulo, Brazil). Two groups (A - community health representatives and $B$ - elderly) participated in this study. Group A was trained using an educational strategy which include digital learning objects, infographics, motivational educational videos, and an interactive learning environment. At the end of the course, these group taught Group B. As a result, after 6-month monitoring, $96.2 \%$ of the Group A participants changed to a better mouth care habit, $84.6 \%$ taught what they had learned, and $65.4 \%$ accomplished activities in their communities. In the Group B, elderly group, 93.2\% learned something new, $83.3 \%$ improved oral health care, and $76.4 \%$ taught someone what they had learned. These digital educational tools and teleducational strategy proved to be efficient. The participants changed habits positively and improved their knowledge on oral health care. The perspective is to use this model in telehealth network for primary care and activities that apply interactive teleducation to diffuse knowledge about oral health care.

Descriptors: elderly, total dental prosthesis, teleducation, oral hygiene. 